Classification

Physics Abstracts

$75.10 \mathrm{H}-05.50$

\title{
Random walks on a closed loop and spin glass relaxation
}

\author{
I. A. Campbell \\ Physique des Solides, Université Paris-Sud, 91405 Orsay Cedex, France
}

(Reçu le 13 septembre 1985, accepté sous forme définitive le 31 octobre 1985)

\begin{abstract}
Résumé. - Les processus de relaxation dans un verre de spin Ising sont discutés en termes de la marche aléatoire sur une surface fermée dans l'espace des phases. Le comportement exponentiel étiré est démontré de façon simple, et la transition verre de spin est associée au seuil de percolation dans l'espace des états propres.

Abstract. - The relaxation processes in an Ising spin glass are discussed in terms of random walks on a closed surface in phase space. Stretched exponential behaviour appears in a simple manner and the spin glass transition is identified with a percolation threshold in eigenstate space.
\end{abstract}

A great deal of interest has been aroused by a "stretched exponential » form of relaxation observed in spin glasses [1]. In impressive large scale simulations on an Ising lattice of $(64)^{3}$ spins coupled by random $\pm J$ interactions, Ogielski [2] has also found relaxations of the correlation function :

$$
q(t)=\left\langle S_{i}(t) S_{i}(0)\right\rangle
$$

which he parametrizes in the form $t^{-x} \exp \left[-(t / \tau)^{\beta}\right]$. As remarked by Ogielski, relaxation in an Ising system is diffusion on the vertices of a hypercube in the spin phase space.

I want to discuss a very simple system - a particle undergoing a random walk on a closed loop. I suggest that this represents some essential aspects of relaxation in complex systems such as a spin glass.

Points on the loop are represented by the angle $\theta$. Suppose random walk steps occur at time intervals $\tau$ and the particle moves through an angle $\pm \delta$ at each step with $\delta \ll \pi$. Initially $\theta=0$, and $I$ will choose to look at the behaviour of the average distance of the particle from its initial position as a function of time,

$$
r=|\overline{\theta(t)}|
$$

Clearly for small $t$, the movement is just a random walk in one dimension, so $r$ increases algebraically, $r \propto t^{1 / 2}$. For very large $t$, the probability distribution for the particle will be close to random, and will be given by :

$$
P(\theta, t)=\frac{1}{2 \pi}(1+\lambda(t) \cos \theta) \text { with } \quad \lambda(t) \ll 1 .
$$


After one further time step,

$$
\begin{aligned}
p(\theta, t+\tau) & =\frac{1}{2}[P(\theta+\delta, t)+P(\theta-\delta, t)] \\
& =\frac{1}{2 \pi}(1+\lambda(t) \cos \delta \cos \theta)
\end{aligned}
$$

i.e. $\lambda(t+\tau)=\lambda(t) \cos \delta$.

This means that the relaxation of the deviation

$$
\Delta r(t)=r(\infty)-r(t)
$$

is exponential in time.

Physically the parameter which separates the two regimes is not so much a time but a distance. Roughly for $r(t) \approx r(\infty) / 2$ the random walk will be much the same as on an infinite lattice; in the other limit $\Delta r(t) \ll r(\infty)$ the remaining deviation will decay exponentially.

We should note one further important point :

$$
\Delta r(t) \propto \exp \left(-\delta^{2} t / 2 \tau\right) \text { at long times . }
$$

The $\delta^{2}$ is because in the situation near random distribution, remaining decay depends on the second derivative of $[P(\theta, t)-P(\theta, \infty)]$ with respect to $\theta$.

Now write $r_{i}(t)=\delta \sqrt{t}$. For short times, $r(t) \propto r_{i}(t)$. For long times, $\Delta r(t) \propto \exp \left(-r_{i}^{2}(t) / 2 \tau\right)$.

It is equally well known that for a particle diffusing on the surface of a sphere, $r \propto t^{1 / 2}$ for small $t$, and $\Delta r(t)$ decays exponentially for $\Delta r \rightarrow 0$ at long times. These limits should hold for diffusion on a closed surface in any dimension, as long as the lattice of available points on the surface is dense.

What happens if the points are not dense ? Alexander and Orbach [3] have discussed diffusion on infinite fractal systems; they find $r \propto t^{1 / 2+\bar{\delta}}$ where $\bar{\delta}$ depends on the system chosen. By analogy with the preceding discussion, for diffusion within a fractal structure on a large closed surface we can expect the following behaviour :

- for small $t$, we will have $r \propto t^{1 / 2+\bar{\delta}}$ as for Alexander and Orbach

- for large $t$ where the system is close to random the analogous expression to $\exp \left(-r_{i}^{2}(t) / 2 \tau\right)$ will be $\exp \left(-\mu t^{2 / 2+\bar{\delta}}\right)$ where $\mu$ is some constant. This is a stretched exponential with $\beta=2 / 2+\bar{\delta}$.

In order to understand this result better, I will work through a simple example. Suppose we have a badly wound ball of string of radius $R$ in $d=3$; the string follows a random path within the ball. Call $l$ a length measured along the string and $r$ the distance from the centre of the ball. For an ant A [4] starting at the centre of the ball and following the string at constant velocity, the average distance from the centre of the ball will increase initially as $r_{\mathrm{A}}(t) \propto \sqrt{l(t)}=\sqrt{t}$ for $l(t) \lesssim R$ and at long times the approach to $r_{\infty}$ will be as $\left[r_{\infty}-r(t)\right] \propto \exp (-l(t) / R)=\exp (-t / \tau)$. The exponential behaviour is not because the number of points on the string is finite but because the string occupies a bounded region of space.

Now suppose a second ant B performs a random walk on the same string. Obviously $l_{\mathrm{B}}(t) \propto \sqrt{t}$. So at short times $r_{\mathrm{B}}(t) \propto \sqrt{l_{\mathrm{B}}(t)} \propto t^{1 / 4}$ and for times such that $l_{\mathrm{B}} \gtrsim R$

$$
\begin{aligned}
{\left[r_{\infty}-r_{\mathrm{B}}(t)\right] } & \propto \exp \left(-l_{\mathrm{B}}(t) / R\right) \\
& \propto \exp \left(-\left(t / \tau_{\mathrm{B}}\right)^{1 / 2}\right) .
\end{aligned}
$$

This stretched exponential behaviour will remain valid as long as $l_{\mathrm{B}}(t)<L$, the overall length of the string. In the « thermodynamic limit » of an infinitely long string the stretched exponential holds for arbitrarily long $t$. 
We can now return to spin glasses. As we have noted the Ising system can be represented by a hypercube. The system will diffuse among those vertices (eigenstates) which are energetically permitted at any given temperature. At high temperature the entropy of the system is high meaning the system has a large number of microstates (eigenstates) available so the diffusion will be on an effectively dense Euclidean lattice. We can expect a parameter representing distance to approach equilibrium exponentially at long times.

As the temperature is lowered the entropy drops, meaning that the number of thermally permitted vertices between which the system diffuses (these are distributed in an irregular way over the hypercube surface because we are in a spin glass) will decrease. At some temperature there will be a cross over from a situation where the system is diffusing on an effectively dense set of vertices to one where the allowed set form a ramified structure on which the system will percolate. To the extent that this ramified lattice can be considered to have a fractal dimension, we can expect $r$ to evolve as $t^{1 / 2+\bar{\delta}}$ for short times and to approach the infinite time value as $\exp \left(-t^{\beta}\right)$ with $\beta=2 / 2+\bar{\delta}$ at long times. Finally lowering the temperature still further the density of available vertices will continue to drop till we reach the percolation threshold. According to Alexander and Orbach, $\bar{\delta}=4$ for any infinite dimension percolating threshold system so at this critical temperature we should expect $r$ to behave as $t^{1 / 6}$ at short times and $\beta$ to be $1 / 3$.

Below this temperature, the system will become non percolating and so non ergodic, i.e. thermally permitted eigenstates of the system having the same energy will no longer be all mutually accessible by relaxation in the thermodynamic limit.

We can compare point by point with Ogielski's results for long times. $\Delta r$ is proportional to $q(t)$ which is a distance on the hypercube. Ogielski finds at high temperature $T>4.5 \mathrm{~J}$ that $q(t)$ behaves exponentially at long $t$. This we identify with diffusion on a dense set of points. For $T<4.5 \mathrm{~J}$, relaxation at long times is of the stretched exponential form with $\beta<1$. This corresponds to percolation on a non-dense set of permitted vertices. Finally when the temperature reaches the glass temperature $T=1.2 \mathrm{~J}$ defined by $\tau \rightarrow \infty$, Ogielski's $\beta$ tends to $1 / 3$ which is just what we could expect at the percolation threshold where $\bar{\delta}=4$ [3].

In conclusion these simple arguments seem to give useful physical insights into the problem of relaxation in an Ising spin glass. The spin glass transition appears as a percolation threshold in eigenstate space, well defined in temperature. It can be shown that this type of ergodicity breakdown is not accompanied by any sharp accident on the specific heat curve, in contrast to a transition with a conventional ordering parameter. When the spin glass transition is approached from high $T$, there should first be a crossover at some higher temperature, corresponding to the eigenstate distribution passing from dense to non-dense; this should be observable experimentally as a change from exponential to stretched exponential relaxation. As the temperature is lowered further and $T_{\mathrm{g}}$ is reached the exponent $\beta$ should tend to $1 / 3$.

These conclusions seem readily generalizable to other homogeneously disordered systems; the scenario which I have just outlined should be fairly robust as the arguments given invoke essentially the disordered character of the system rather than any characteristics specific to Ising magnets. This may explain why the stretched exponential relaxation is ubiquitous in disordered systems $[1,5]$.

\section{Acknowledgments.}

I would like to thank G. Toulouse and R. Jullien for enlightening discussions. 


\section{References}

[1] Chamberlin, R. V., Mozurkewich, G. and Orbach, R., Phys. Rev. Lett. 52 (1984) 867.

[2] OGIELSKI, T. A., to be published.

[3] Alexander, S. and Orbach, R., J. Physique Lett. 43 (1982) L-625.

[4] De GenNes, P. G., La Recherche 7 (1976) 919.

[5] JONSCHER, A. K., Nature 267 (1977) 673.

NGAI, K. L., Comments on Solid State Physics 9 (1979) 127.

Chamberlin, R. V., J. Appl. Phys. 57 (1985) 3377. 